\title{
Initial Tests of an AC Dipole for the Tevatron
}

\author{
R. Miyamoto*, A. Jansson ${ }^{\dagger}$, S. Kopp*, and M. Syphers ${ }^{\dagger}$ \\ *The University of Texas at Austin, Department of Physics, Austin, Texas 78712 \\ ${ }^{\dagger}$ Fermi National Accelerator Laboratory, Batavia, Illinois 60510
}

\begin{abstract}
The AC dipole is a device to diagnose transverse motions of a beam. It can achieve large-amplitude oscillations without two inevitable problems of conventional kicker/pinger magnets: decoherence and emittance growth. While not the first synchrotron to operate with an AC dipole, the Tevatron can now make use of its recently upgraded BPM system, providing unprecedented resolution for use with an $\mathrm{AC}$ dipole, to measure both linear and nonlinear properties of the accelerator. Plans are to provide AC dipole systems for both transverse degrees of freedom. Preliminary tests have been done using an audio power amplifier with an existing vertical pinger magnet, producing oscillation amplitudes up to $2 \sigma$ at $150 \mathrm{GeV}$. In this paper, we will present the configuration of this system. We also show the analysis of a first few data sets, including the direct measurement of beta functions at BPM locations.
\end{abstract}

Keywords: AC Dipole, Lattice Measurement.

PACS: 29.27.-a, 41.85.-p

\section{INTRODUCTION}

\section{Motivation}

The AC dipole is a tool to study transverse motions of a beam in a synchrotron. As kicker/pinger magnets which have been conventionally used to make measurements of beam properties, the AC dipole also creates transverse oscillations with large amplitudes but it is done by driving the beam instead of giving a single turn kick. As stated in [1] and [2], the AC dipole has two advantages over the kicker/pinger magnets. First, the signal of the oscillation created by the AC dipole does not decohere. This is particularly important in measurements of nonlinear properties of a beam. Second, there is no significant emittance growth after the usage of the AC dipole and, hence, beam diagnosis based on the $\mathrm{AC}$ dipole can be done even during usual operations of a synchrotron. These properties of the AC dipole have been proven through previous experiments in AGS and RHIC at BNL and in SPS at CERN [3], [4], and [5].

The BPM system of the Tevatron has been recently upgraded and its resolution is now as low as $20 \mu \mathrm{m}$. With this BPM system, the AC dipole is expected to make it possible to measure both linear and nonlinear properties at an unprecedented level. Hence, the plan is to install the AC dipole systems for both transverse degrees of freedom. 
For the Tevatron, there are three identical pinger magnets which can be potentially used as AC dipoles. One is used as a vertical pinger and the other two are its spares. Tests have been done with these pinger magnets and a temporary audio amplifier [6] to check that these magnets can work properly as AC dipoles.

\section{Driven Coherent Oscillation}

The magnetic field created by an AC dipole changes sinusoidaly in time. Under the influence of such dipole field, a beam performs driven coherent oscillations. The transverse (either horizontal or vertical) position change due to this oscillation is expressed by the following equation [1], [2], and [3]:

$$
z_{ \pm}^{(m)}(s+n C) \cong \frac{B_{m} L}{4 \pi(B \rho) \delta_{ \pm}} \sqrt{\beta_{m} \beta(s)} \cos \left( \pm 2 \pi v_{m} n+\Delta \psi(s)+\chi_{ \pm}\right),
$$

where $s$ is the longitudinal position in the ring, $n$ is the number of turns after the AC dipole is turned on, $C$ is the circumference of the ring, $B_{m} L$ is the integrated magnetic field of the dipole defined as the magnetic field times the length of the magnet, $(B \rho)$ is the magnetic rigidity, $\beta(s)$ and $\beta_{m}$ are beta functions at $s$ and the longitudinal position of the AC dipole, $v$ and $v_{m}$ are the natural tune and tune of the AC dipole, $\Delta \psi(s)$ is the phase advance from the position of the AC dipole to $s$, and $\chi_{ \pm}$is a constant phase. The driven coherent oscillation is excited when the fractional part of $v_{m}, \delta v_{m}$, is close to the fractional part of the natural tune $\delta v$ or $1-\delta v$. The \pm sign in Eq. (1) corresponds to these two modes and $\delta_{+}$and $\delta_{-}$are defined by $\delta_{+} \equiv \delta v-\delta v_{m}$ and $\delta_{-} \equiv(1-\delta v)-\delta v_{m}$.

\section{Emittance Preservation by Adiabatic Ramps}

In its operation, the amplitude of the magnetic field created by an AC dipole is first slowly increased, second kept at the maximum during measurements, and finally slowly decreased to zero. In our tests, the amplitude was modulated by the trapezoidal envelope function with linear ramp up and ramp down.

The simplest model estimates the increase of the transverse beam size after one cycle of the AC dipole operation is proportional to $B_{m} L /\left(\delta^{2} n_{r}\right)$, where $n_{r}$ is the number of turns to ramp up and ramp down [7]. Hence, the adiabatic processes of slow ramp up and ramp down guarantee the preservation of the emittance.

\section{SYSTEM CONFIGURATION}

Fig. 1 is a schematic diagram of the AC dipole system for the Tevatron. The magnet is the only element inside the tunnel and it is connected to other elements through two parallel cables. The power supply is an audio amplifier which amplifies the signal created by two arbitrary waveform generators. The parallel capacitor is making a resonant circuit with the magnet but the series capacitor is off resonance and just removing noise form the amplifier. The current passing through the magnet is monitored by a current transformer (CT). 


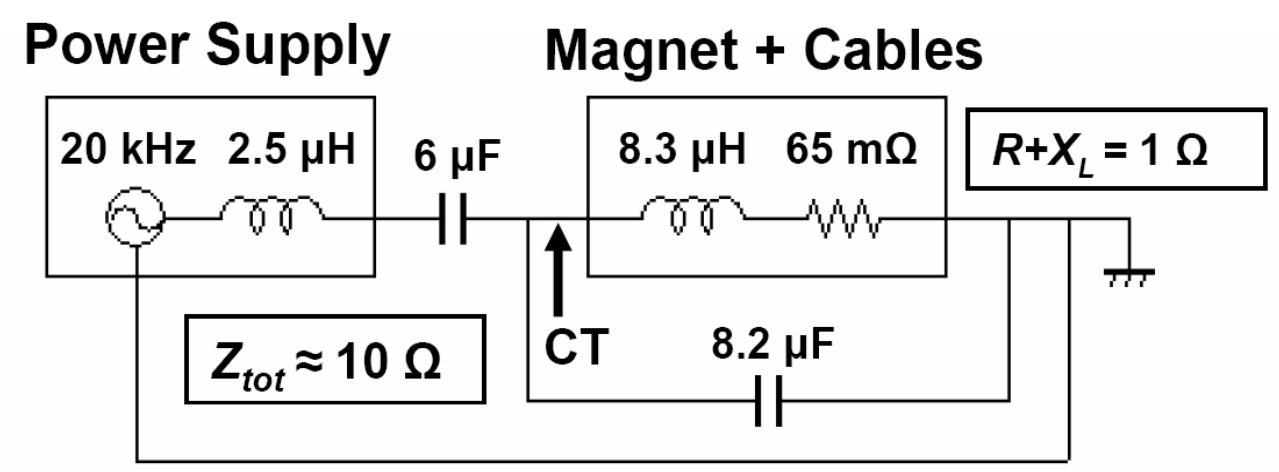

FIGURE 1. The schematic diagram of the AC dipole system for the Tevatron.

\section{Frequency}

Frequency of an AC dipole is determined by the tune and revolution frequency of a synchrotron. So far, tests have been done only for the vertical degree of freedom at the injection energy of $150 \mathrm{GeV}$. At injection, the vertical tune of the Tevatron is 20.578 and so $\delta v_{m}$ must be close to either $\delta v(0.578)$ or $1-\delta v(0.422)$. For our system, the smallest value of $0.422-\delta_{-}$is chosen for $v_{m}$. Since the revolution frequency of the Tevatron is $47.712 \mathrm{kHz}$, the frequency of our AC dipole is $47.712 \mathrm{kHz}$ multiplied by $0.422-\delta_{-}$. For the typical $\delta_{-}$of -0.01 , it is $20.612 \mathrm{kHz}$.

\section{Magnet and Cables}

The vertical pinger magnet used for our initial tests is a single turn iron core magnet with a ceramic pipe. Since its length and gap width are known as $2 \mathrm{~m}$ and $5 \mathrm{~cm}$, the relation between the current $I_{m a g n e t}$ and integrated magnetic field $B_{m} L$ can be estimated:

$$
B_{m} L \cong 0.5 I_{\text {magnet }} \mathrm{Gm} \text {. }
$$

The magnet is connected to other elements of the system on the surface level through two parallel RG-220 coaxial cables of $22 \mathrm{~m}$ length. Hence, the inductance and resistance of these cables must be added to those for the magnet. The capacitance of cables can be ignored for this cable length and frequency since their capacitative reactance is larger than their inductive reactance by a factor of $10^{3}$. The total inductance and resistance of the magnet and cables were measured and they were 8.3 $\mu \mathrm{H}$ and $65 \mathrm{~m} \Omega$. At $20 \mathrm{kHz}$, the impedance of the combination is $1.0 \Omega$.

\section{Arbitrary Waveform Generators}

The input signal to the audio amplifier is created by mixing signals from two arbitrary waveform generators. One of the generators (Stanford Research DS 345) creates sine wave of about $20 \mathrm{kHz}$ and the other one (Agilent 33250A) modulates its amplitude by the trapezoidal envelope function which is discussed in the previous section. The upper graph in Fig. 2 is an example of the input signal. 


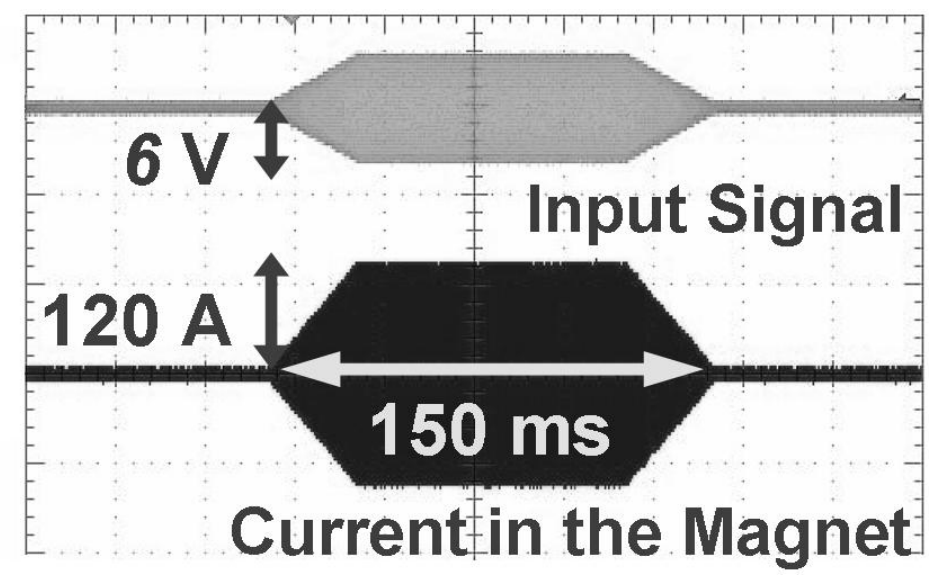

FIGURE 2. Upper: the signal created by two arbitrary waveform generators supplied as input to the amplifier. Lower: the current in the AC dipole measured by the current transformer (CT) in Fig. 1. The horizontal axis is time and one division is $20 \mathrm{~ms}$ which corresponds to approximately 1,000 revolutions of the beam in the Tevatron. One full ramp up (20 ms), excitation period (100 ms), and ramp down (20 $\mathrm{ms}$ ) of the magnet is shown.

\section{Power Supply}

The power supply used in our tests is Crown M-600 audio amplifier. From the manual of the amplifier [6], the relation between the maximum output voltage and the load impedance is given by Fig. 3. When the load impedance is lower than $4 \Omega$, although there is no data for $20 \mathrm{kHz}$ in the manual, the curve for $20 \mathrm{kHz}$ is expected to go under that for $1 \mathrm{kHz}$. Notice more voltage can be produced when the impedance gets larger but the amount of increase gets much less once the impedance surpasses around $5 \Omega$. Another important property of the amplifier is its current limit. According to the manual, it is about $20 \mathrm{~A}$.

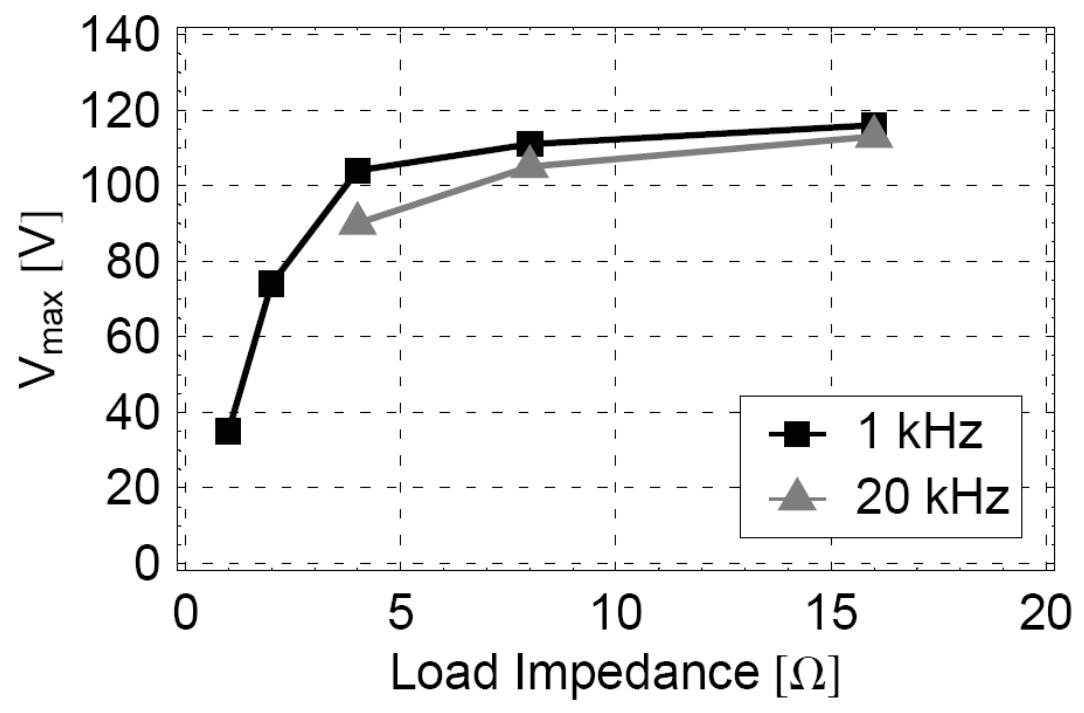

FIGURE 3. The relation between the output voltage and load impedance for the Crown M-600 audio amplifier. For our resonant circuit (Fig. 1), the total impedance is $Z_{t o t} \sim 10 \Omega$ and the excitation frequency is $f \sim 20 \mathrm{kHz}$. 


\section{Parallel Resonant Circuit}

As previously discussed, the magnet and cable is equivalent to an inductor and a resistor. A power supply can drive such a system either directly or with help of a capacitor by forming a series or parallel resonant circuit. A series resonant circuit helps if the power supply is capable of producing large current but limited in voltage. On the contrary, a parallel resonant circuit suits for the power supply with large voltage and limited current. The Crown audio amplifier can produce more than $100 \mathrm{~V}$ (Fig. 3) but its current is limited to 20 A. Hence, it is natural to adopt a parallel resonant circuit to maximize the current in the magnet. It was also checked that the direct drive and series resonant circuit could produce only about $20 \mathrm{~A}$ of current.

Fig. 4 is the simulation of the total impedance when an $8.2 \mu \mathrm{F}$ capacitor is connected to the magnet and cables in parallel. The impedance is roughly $10 \Omega$ around $20 \mathrm{kHz}$ and the power supply produces more than $100 \mathrm{~V}$ for $10 \Omega$ (Fig. 3). Since this $100 \mathrm{~V}$ directly applies to the magnet and cables with $1 \Omega$ of impedance (Fig. 1), more than $100 \mathrm{~A}$ of current is expected. The agreement of this estimation with a measurement can be seen in the lower graph of Fig. 2. It shows the current through the magnet measured by the current transformer (CT) is about $120 \mathrm{~A}$ at $20 \mathrm{kHz}$.

The internal inductance of the power supply and other capacitor removing the noise of the power supply add up to impedance of only about $1 \Omega$. Since this is much smaller than the impedance of the parallel section, the most of voltage created by the power supply applies on the parallel section.

Since the impedance at $20 \pm 2 \mathrm{kHz}$ is about $5 \Omega$ (Fig. 4), the output voltage at those frequencies is about $95 \mathrm{~V}$ which is roughly $80 \%$ of the maximum (Fig. 3). Therefore, besides the typical roll-off of the audio amplifier over $20 \mathrm{kHz}$, the system can maintain $80 \%$ of output within the range of $\pm 2 \mathrm{kHz}$ which corresponds to \pm 0.04 of $v_{m}$. In contrast to the AC dipole system for the RHIC which has a high Q magnet and spoiler [8], our system naturally has low Q.

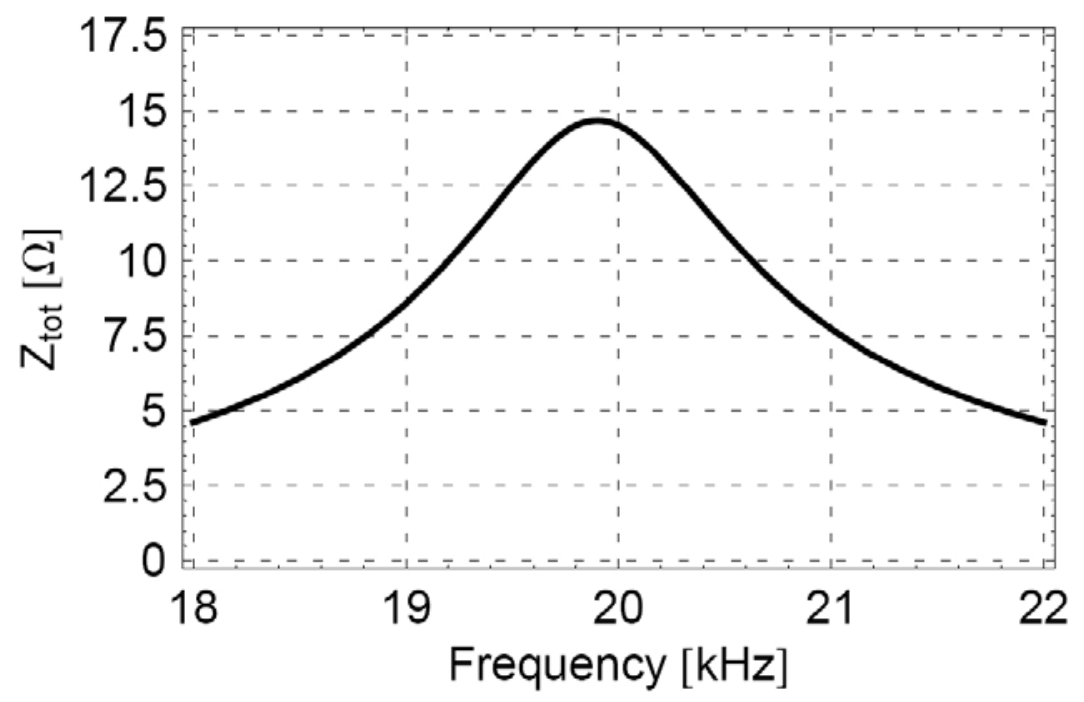

FIGURE 4. The total impedance of the magnet, cables, and parallel capacitor around $20 \mathrm{kHz}$. From the figure, $\mathrm{Q}$ of the circuit is roughly 10, however, frequency dependence of the system itself is less as explained in the text. 


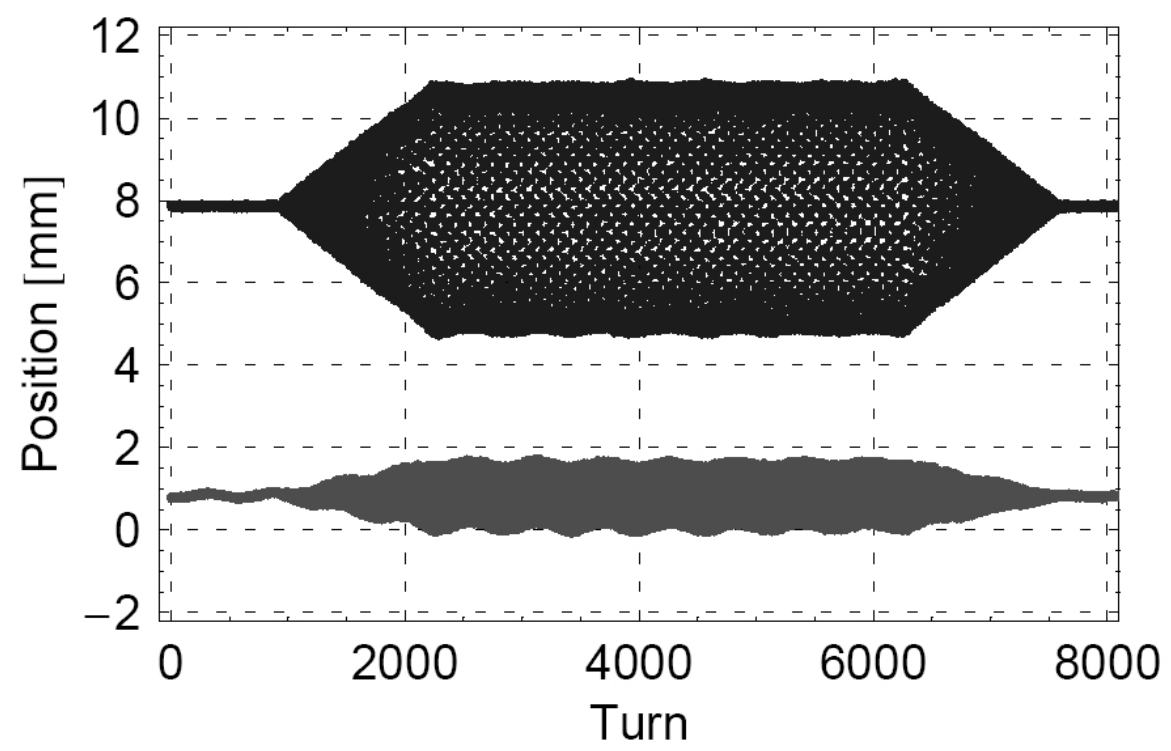

FIGURE 5. Turn-by-turn BPM data of the beam excited by the vertical AC dipole. The upper is the vertical and lower is the horizontal motions. The locations of the BPM's are at downstream of the A0 straight section of the Tevatron. The beam was the uncoalesced single-bunch at the injection energy.

\section{TEST RESULTS AND AN EXAMPLE OF BEAM DIAGNOSIS}

\section{Turn-by-turn BPM Data}

Fig. 5 is an example of a turn-by-turn beam position data when the beam is excited by the AC dipole. It is possible to see the vertical beam motion is following the shape of the input signal in Fig. 2.

For the data in Fig. 5, the expected amplitude from Eq. (1) and (2) is about $5 \mathrm{~mm}$ but only about $3 \mathrm{~mm}$ is measured. The oscillation amplitudes were always observed to be 30 to $40 \%$ lower than expectations in our initial tests. The data indicates there was strong coupling in the machine when the data was taken. Also, the parameters of the magnet are not yet completely known since an existing magnet was used. There are other possible reasons for the shortfall in observed amplitudes and the conclusion has not been made yet.

\section{$\delta$ Dependence of the Driven Coherent Oscillation}

The amplitude of the driven coherent oscillation excited by the AC dipole is roughly proportional to the inverse of $\delta_{ \pm}$[1], [2], [3], and [5]. Fig. 6 shows the measured amplitudes for different $\delta_{-}$with a fixed magnetic field. Although the amplitude is smaller than expected as before, data fit well to the reciprocal of $\delta_{-}$. 


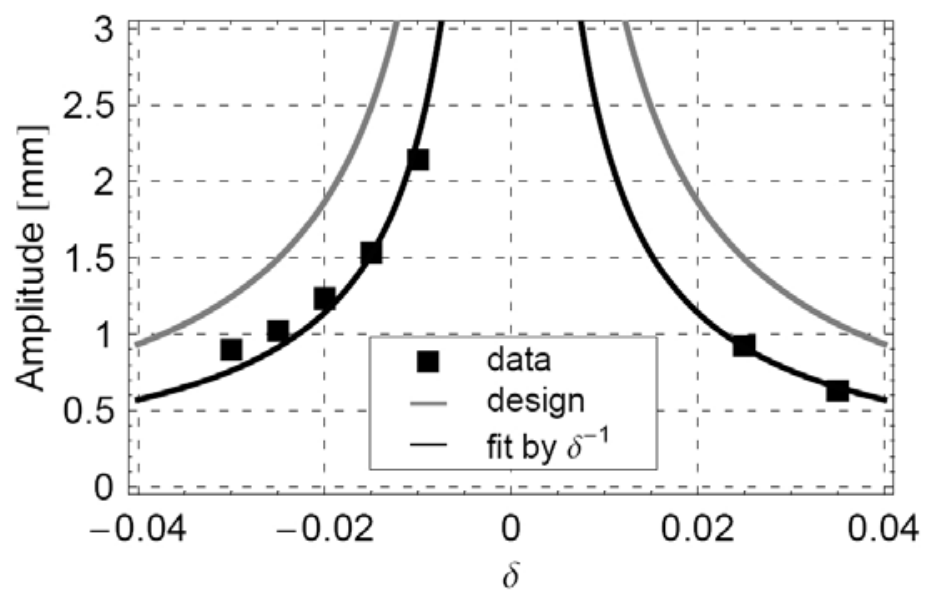

FIGURE 6. $\delta_{-}$dependence of the amplitude of the driven coherent oscillation. The gray line (upper) is the expected amplitudes from the dimensions of the dipole magnet. The black is the fit to data by the reciprocal of $\delta_{-}$.

\section{Emittance Preservation}

In our initial tests, a flying wire in section E11 of the Tevatron was used every time before and after the AC dipole was fired to record the change in the beam size. According to [7], the beam size increase is proportional to $B_{m} L / \delta_{-}{ }^{2}$ when times for ramp up and ramp down are fixed. In Fig. 7, changes in square of the beam size before and after the excitations of the AC dipole was plotted as a function of $B_{m} L / \delta_{-}{ }^{2}$.

Negative changes in Fig. 7 indicate the flying wire itself has uncertainty. For the most of measurements, changes were within this range and, hence, beam sizes were preserved within the measurement uncertainty. This result is consistent with earlier observations [3] and [5].

From this data set, no obvious correlation is seen between the beam size increase and $B_{m} L / \delta_{-}{ }^{2}$. One of the possible reasons is the ramp times are too large to see this correlation. Further study and measurements are required to have more detailed understanding about the beam size growth induced by the AC dipole.

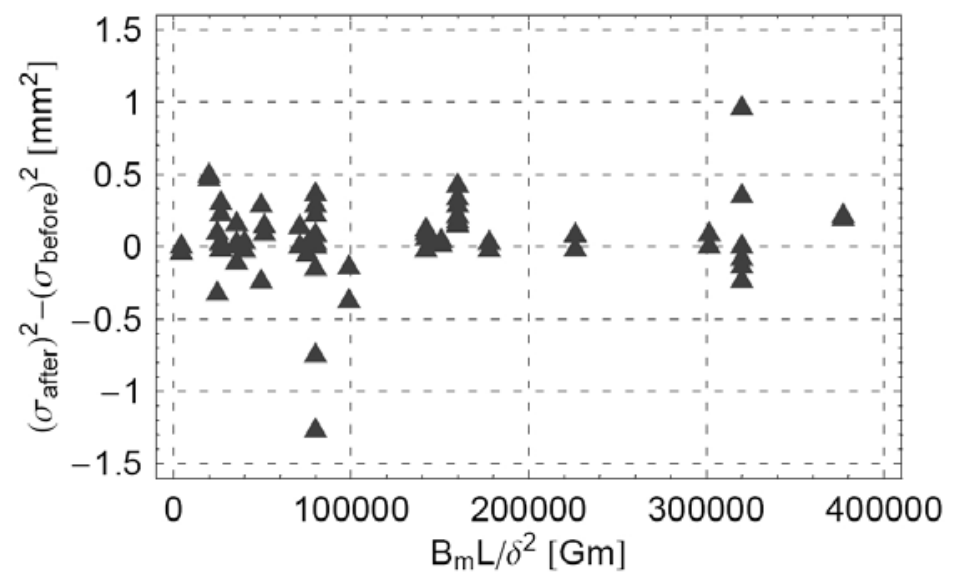

FIGURE 7. Increases in square of the transverse beam size after excitations of the AC dipole as a function of $B_{m} L / \delta_{-}{ }^{2}$. 


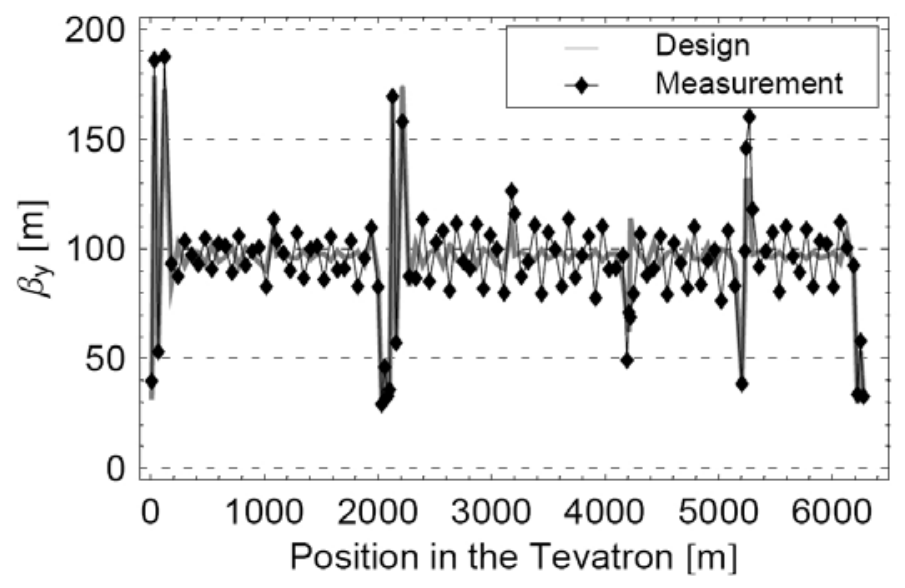

FIGURE 8. Comparison of the design and measured vertical beta functions at locations of vertical BPM's in the Tevatron. 0 and 2,100 $\mathrm{m}$ are two interactions points of the Tevatron: $\mathrm{B} 0$ and $\mathrm{D} 0$.

\section{Beta Function Measurement}

Linear optics measurement is an example of beam diagnostic using the AC dipole. From Eq. (1), the amplitude of the driven coherent oscillation at $s$ is proportional to the square root of the beta function at the point. Hence, the relative values of beta functions at each BPM can be calculated by comparing the squares of the oscillation amplitudes measured at each BPM's. There are a few ways to decide the exact value of the beta function at a point in the ring from the BPM data of the AC dipole [9]. In this analysis, the average of squares of amplitudes at each BPM was simply scaled to the average of the design beta functions

Fig. 8 shows the comparison of the design and measured beta functions of the injection energy at vertical BPM locations in the Tevatron. Although the measurement is roughly following the design, the sinusoidal deviation along the ring (beta beating) can be observed. Fig. 9 shows the relative differences between the design and measured beta functions around the Tevatron. If there are two sources of quadrupole errors, the amplitude of the beta beating may change at these two points. Hence, Fig. 9 suggests two sources are around 100 and 2,100 m which are very close to the two interaction points and their final focus triplet quadrupoles.

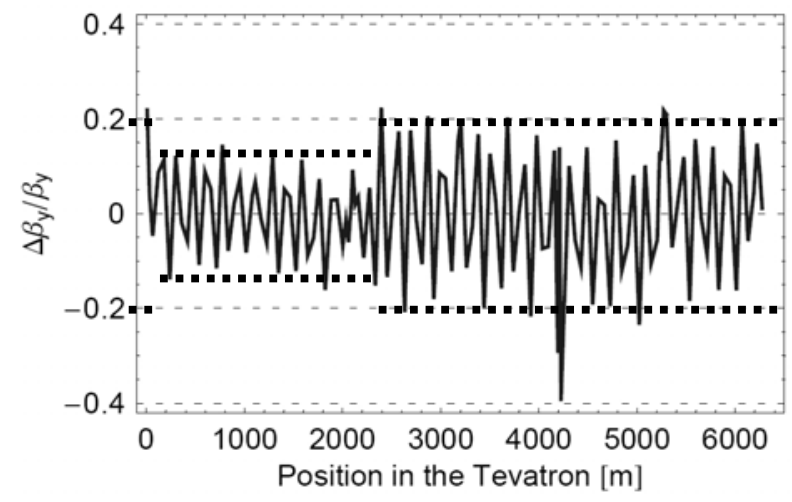

FIGURE 9. The beating, $\left(\beta_{\text {measurement }}-\beta_{\text {design }}\right) / \beta_{\text {design }}$, of the vertical beta functions at locations of vertical BPM's in the Tevatron. 


\section{CONCLUSIONS}

Initial tests of the $\mathrm{AC}$ dipole for the Tevatron have been done by using existing pinger magnets and an audio amplifier. Clean driven coherent oscillations were observed. The amplitude of $2.5 \mathrm{~mm}$, which is about twice of the natural beam size, was achieved. Basic properties of the device were checked, such as the $\delta$ dependence of the amplitude and emittance preservation. With the BPM data of the driven coherent oscillations, preliminary linear optics measurements were also done.

In the future, some properties of the magnets such as field strength and quality must be measured with reasonable precisions. An upgrade of the power supply is necessary to reach enough oscillation amplitude to study nonlinear properties of the Tevatron. Another AC dipole for the horizontal direction is also planned.

\section{ACKNOWLEDGMENTS}

The authors would like to thank the following people of Fermilab for their support

of our project: Dan Bollinger, Bob Hively, Chris Jensen, Vahid Ranjbar, Cheng-Yang Tan, Roger Tokarek, and Dan Wolff.

\section{REFERENCES}

1. S. Peggs, "Nonlinear Diagnostics Using AC Dipoles" in Proceedings of the 1999 Particle Accelerator Conference, New York, NY, edited by A. Luccio and W. MacKay (IEEE, Piscataway, NJ, 1999), pp. 15721574.

2. S. Peggs and C. Tang, BNL Report No. RHIC/AP/159, 1998.

3. M. Bai et al., Phys. Rev. E 56, 6002-6007 (1997).

4. M. Bai el al., "RHIC Vertical AC Dipole Commissioning" in Proceedings of the European Particle Accelerator Conference, Paris, 2002, edited by T. Garvey et al. (EPS-IGA and CERN, Geneva, 2002), pp. 1115-1117.

5. O. Berrig et al., "Excitation of Large Transverse Beam Oscillations Without Emittance Blow-up Using the ACDipole Principle" in Proceedings of the $5^{\text {th }}$ European Workshop on Beam Diagnostics and Instrumentation for Particle Accelerators, Grenoble, France, 2001, edited by K. Scheidt et al. (ESRF, Grenoble, France, 2001), pp. 82-84.

6. Instruction Manual M-600 Audio Amplifier, (Crown International Inc., Indiana).

7. R. Tomas, Phys. Rev. ST Accel. Beams 8, 024401 (2005).

8. M. Bai et al., "RHIC AC Dipole Design and Construction" in Proceedings of the 2001 Particle Accelerator Conference, Chicago, IL, edited by P. Lucas and S. Webber (IEEE, Piscataway, NJ, 2001), pp. 3606-3608.

9. M. Bai, "Beam Manipulation with an RF Dipole" in Proceedings of the 1999 Particle Accelerator Conference, New York, NY, edited by A. Luccio and W. MacKay (IEEE, Piscataway, NJ, 1999), pp. 387-391. 